\title{
Mobile Ad-Hoc Network Data Communication in Large Geographic Areas
}

\author{
Leslie D. Fife \\ School of Computing \\ Brigham Young University Hawaii \\ Laie, HI 96762, USA \\ ldfifedcs.byuh.edu
}

\author{
Le Gruenwald \\ School of Computer Science \\ University of Oklahoma \\ Norman, OK 73019, USA \\ ggruenwaldeou.edu
}

\begin{abstract}
A Mobile Ad-Hoc Network (MANET) is a group of wireless, mobile, battery-powered clients and servers that autonomously form temporary networks. Three data communication modes can be provided in a MANET: data broadcast, data query, and peer-to-peer messaging. In support of the data communication needs existing in these networks, a database is utilized for data broadcast and data query. This paper presents the TriM protocol (Tri-Modal communication) to handle data communication in a MANET database system. TriM accommodates node disconnection and reconnection through periodic synchronization while having minimum power consumption. This protocol has been shown capable of allowing all three data communication modes in a single network deployment in small geographic regions. This paper extends those results by evaluating TriM in the larger geographic regions present in the military scenario. Simulation shows that TriM is capable of providing poweraware and mobile-aware data communication services in the larger regions of the military scenario.
\end{abstract}

\section{INTRODUCTION}

A MANET is a collection of mobile, wireless and battery powered servers and clients. The topology of a MANET changes frequently as nodes move. A MANET is a potential solution whenever a temporary network is needed and no fixed infrastructure exists. An example application is the military scenario, which includes a large number of nodes moving over terrain without the benefit of a fixed power grid or wired network topology.

MANETs differ from traditional mobile networks. In traditional mobile networks the servers, and potentially some clients, are stationary and powered by a fixed power grid. In addition, servers and some clients may be connected over a wired network. The servers communicate with the mobile clients over a wireless link. A MANET provides the traditional wireless network capabilities of data push and data pull as well as allowing clients to communicate directly in peer-to-peer communication without the use of a server, unless necessary for routing . Due to servers having a larger capacity than clients [7], we assume that servers contain the complete database management system (DBMS) and bear the responsibility for data broadcast and satisfying client queries.

Nodes (clients and servers) may not remain connected to the MANET throughout their life. To be connected to the network, a node must be able to hear the transmission of at least one other node on the network and have sufficient power to function. We assume a fixed transmission power level. Network nodes (clients and servers) may operate in any of the three modes that are designed to facilitate the reduction in power used. These are: transmit - this mode uses the most power, allowing transmission and reception of messages, receive - this mode allows the processing of data and reception of transmissions, and standby - in this mode, the CPU does no processing, transmitting or receiving.

Traditional mobile network research must address the limitations of the wireless bandwidth as well as the mobility and battery power of clients. MANET must consider these issues for both clients and servers. This prevents the use of current traditional mobile network data communication protocols, which assume stationary servers with unlimited power.

The majority of research in MANET has centered on routing issues [1][9]. Over the past few years, interest in data communication has been increasing [7][17][18]. However, most current MANET data communications protocols have provided only one or two modes of data communication. TriM, for Tri-Modal communication, is a new protocol that has been designed specifically for the MANET environment. A complete description of the TriM protocol can not be included here but can be found at [6]. TriM has shown through simulation to be capable of providing the ability for a MANET to use all three modes of data communication in a small $1 \mathrm{~km} \mathrm{x} 1 \mathrm{~km}$ region [5]. These simulation results were encouraging. For this reason, this paper extends the previous work by evaluating TriM in the much larger military scenario $(10 \mathrm{~km} \mathrm{x} 15 \mathrm{~km})$ and analyzing the effects of geographic region's size on TriM's performance.

\section{CURRENT MANET DATA COMMUNICATION RESEARCH}

The data communication research issues in MANET center around two areas. These issues are described in detail in [4][6]. The first area concerns the limitations of the environment (wireless, limited bandwidth, battery powered, mobile) for both clients and servers. The second area concerns the three ways in which MANET data communication may take place. Within this area, concerns due to data push, data pull and peer-to-peer communications exist.

Some work in MANET data communication has been scenario specific. In the work of Jung [11], location dependent queries in urban areas are addressed. Tang [14] 
adapts MANET data broadcasting to power controlled wireless ad-hoc networks. In these networks, servers have the ability to broadcast at one of several discrete power levels. The work of Tseng [15] deals with the broadcast storm.

Wieselthier, et al, have been working on MANET data broadcast. Their approach is the construction of a minimum-energy tree rooted at the broadcast source. Two algorithms, one for broadcast and one for multicast were described.. The algorithms were tested and showed that by utilizing broadcast in a mobile environment, energy savings can be achieved. However, the networks tested were small and node mobility was not addressed. The cost of building the tree is considered negligible by the authors. However, it has been shown that tree-based protocols do poorly when there is node mobility The problems of limited bandwidth, the need for tree maintenance, and node mobility also remain.

Two protocols to handle data push and a limited form of data pull within the MANET were proposed in . They use a global network where all servers in a region know the location and power of all other servers in the region and full replication of the database is assumed. Periodically, each server broadcasts its location and power level . Data deadlines are used to determine which data requests to service. The protocols include a leader selection protocol. The leader coordinates the broadcast responsibilities of other servers in its region by determining which portion of the broadcast each server transmits. Between broadcast transmissions, clients are permitted to query the servers . These algorithms have a potentially large overhead where less popular items may starve or be broadcast too late while awaiting leader selection .

The second protocol includes the use of a popularity factor (PF), as suggested by Datta . The PF is a measure of the importance of a data item. The PF increases each time a request is made for a data item . An additional factor, Resident Latency (RL) also affects the PF. If it has been too long, the need to broadcast the item may be gone. RL is system and scenario specific. The PF decreases whenever request age exceeds the $\mathrm{RL}$. To localize data delivery, the lead server assigns each server the amount of data, but not which items, to broadcast . In addition to leader selection costs, calculation of the PF and comparison to the RL add to the overhead.

\section{TriM DATA COMMUNICATION PROTOCOL}

In the following subsections, the TriM data communication protocol is presented. Due to space limitation, a complete treatment of the protocol including its specific parameters is not provided here, but can be found in [6]. Figure 1 shows the four stages in a single iteration of TriM. The protocol will cycle through these stages repeatedly. Prior to the first iteration of the protocol, the network is initialized by setting all protocol parameters.

Data communication can take place in the data push stage and the data pull stage. The synchronization stage allows servers/clients to synchronize and detect the other nodes in their immediate vicinity. The idle stage allows the setting of a period of time during which all nodes are inactive. This effectively determines the frequency of data broadcast. The service cycle repeats until the network is taken out of service or all nodes fail.

\begin{tabular}{|l|c|c|c|}
\hline $\begin{array}{l}\text { Synch } \\
\text { Stage }\end{array}$ & Data Push & Data Pull Stage & Idle \\
& Data & Data Request & Stage \\
& Broadcast & Peer-to-Peer & \\
\hline
\end{tabular}

Fig. 1. TriM Data Communication Protocol

\section{A. Network Initialization and Control}

There are four stages, synchronization, data push, data pull and idle, in TriM. The first three are active while the last one is inactive. Within each active stage there are tasks associated with the servers and tasks associated with the clients.

Network initialization is accomplished when deploying a MANET. The network designer determines the length of each of the network stages according to the needs of the network and the characteristics of that particular deployment. Network initialization involves a variety of parameters. Each node in the network (server and client) is initialized using the same parameter values. These values are static throughout the MANET deployment. The database maintained by the servers is assumed to be fully replicated. Each server and client independently monitors time and uses this to synchronization with other nodes.

\section{B. TriM Synchronization Stage}

The synchronization stage has two parts. The first part is restricted to the transmission of information by servers. Servers transmit their unique ID and location. This information is necessary to perform peer-to-peer message routing and is used by clients during data query to select the nearest server to query. The individual presence of servers is critical to the protocol. Sufficient time is allocated during server synchronization to allow all servers to transmit their information independently. Each server knows the number of servers that were deployed during network initialization. The unique IDs are numbered from 1 to $n$. Each server transmits its information in turn, waiting the appropriate period of time before transmitting its information. The importance of the server information to the protocol prohibits transmission in parallel. Collisions in the limited bandwidth of wireless networks could cause the loss of critical information from neighboring servers. The amount of time a server must wait is determined by the number of servers having smaller IDs and the time needed for it to transmit its ID and location.

The second stage is for transmission of information by clients. Each client transmits its unique ID and location. To perform routing of peer-to-peer messages during the data pull stage, the location of each client is needed. However, the number of clients is potentially large and it may not be possible to reserve sufficient time for each client to 
transmit independently. Clients transmit their information when the transmission channel is clear.

By regularly synchronizing all nodes, each node will be in the same protocol stage at the same time. This prevents contention over the limited network bandwidth. This is especially important during data broadcast, which immediately follows synchronization. The results of synchronization also play a role in data query and peer-topeer communication. During synchronization, nodes can determine if they are currently disconnected from the network. If a node detects no other nodes during synchronization, it will sleep until the next service cycle.

\section{TriM Data Push Stage}

The second stage of the service cycle is the data push stage. The data push stage occurs before data pull so that the maximum number of potential data needs can be served at the lowest power cost. Separating data push and data pull reduces the contention for the limited bandwidth. When the data needs of a client are satisfied by the broadcast, the need for data query is reduced.

Servers - Data Push Stage: The autonomous and mobile nature of this self-organizing network suggests independent servers. The decision to transmit a data broadcast is a local one. The contents of the broadcast are also partially determined by each server. The data broadcast will be composed of both a pre-selected set of data items and a set of dynamically selected items. The preselected items are determined at MANET deployment by the network designer. These are data items that each client needs frequently. The fixed portion of the broadcast is the same for each server. The dynamic portion of the data broadcast will vary, depending on the unserved data queries from the previous service cycle.

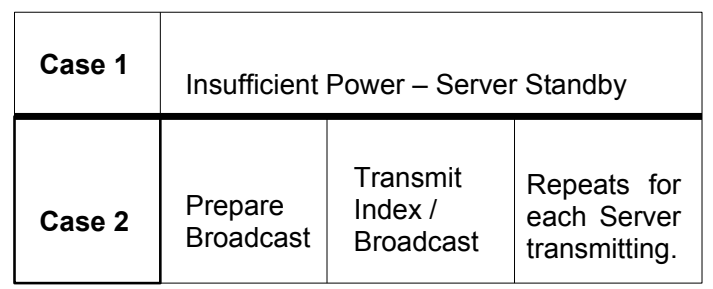

Fig. 2. TriM Data Push Stage - Server

\begin{tabular}{|l|l|l|}
\hline Case 1 & \multicolumn{2}{|c|}{ No Servers - Standby } \\
\hline & $\begin{array}{l}\text { Standby } \\
\text { while } \\
\text { broadcast } \\
\text { prepared }\end{array}$ & $\begin{array}{l}\text { Listen to index } \\
\text { transmission for all } \\
\text { servers detected. } \\
\text { Listen to data as } \\
\text { needed, Standby } \\
\text { otherwise. }\end{array}$ \\
\hline
\end{tabular}

Fig. 3. TriM Data Push Stage - Client

Figure 2 shows the broadcast portion of the service cycle for servers. Two possible situations are shown. In the first situation, the server has insufficient power to transmit an index and data broadcast. In the other case, the index and broadcast are transmitted in server ID order with each server being allocated a broadcast slot to prevent collision.
As the size of the MANET broadcast is meant to be of minimal size, a single transmission of the index is preferred as transmission of the index takes time and consumes power. As several servers may broadcast in the same region, duplication of the broadcast static portion is a waste of power. To some extent, this cannot be prevented. A client may be in the transmission range of several or only one of the servers, depending on its geographic location.

Clients - Data Push Stage Clients, like servers, have two potential situations during data push. If a client detects no servers during synchronization, it will be in standby. The client behavior is shown in Figure 3. Each client knows from the synchronization stage which servers will transmit in their region. The clients can then tune into receivable transmissions. The client need only listen to the static portion once. A client will also check the index for any needed dynamic data items. It will use the index to determine when the data item will be transmitted. A client needs only listen to transmitted indices, the static data portion once and dynamic data items of interest. To listen to these items, the client must be in receive mode.

\section{TriM Data Pull Stage}

During the data pull stage, both data query and peer-topeer communication occur. Servers both respond to data requests from clients and perform any requested peer message routing. In data query, clients request data from servers. In peer-to-peer communication, clients communicate directly with other clients. Selection of peerto-peer communication partners is determined by the application rather than TriM. If the client contacted is disconnected from the network, the message is dropped. Clients will only transmit the same query or peer message once during a single service cycle.

Servers - Data Pull Stage The actions of servers during the data pull stage of the service cycle are shown in Figure 4. The servers have two primary tasks during the data pull stage. First they must respond to data queries. Second, servers must route client peer communications when requested.

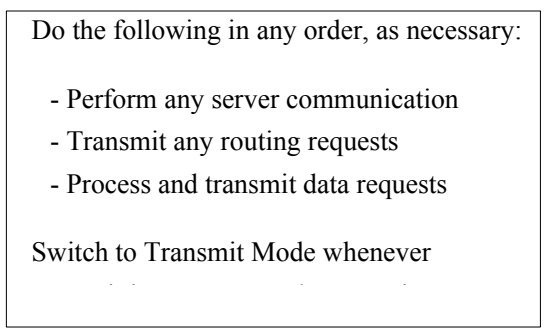

Fig. 4. Trim Data Pull Stage - Server

Transmit while transmitting queries to servers or responses to clients.

Receive while generating queries, receiving results of query from server or messages from other clients 
Fig. 5. TriM Data Pull Stage - Client

Clients - Data Pull Stage A SMH has only a few potential tasks during data pull as shown in Figure 5. The first situation is when a client needs to make a data request. Second, a client may need to communicate directly with another client. If the target client is detected during the most recent synchronization stage, it will transmit to the target directly. Otherwise, a routing request will be sent to a server. Finally, a client may receive a peer message.

\section{E. TriM Idle Stage}

Following the data pull stage a MANET will enter into a period where all nodes are in standby. The length of this period is determined by the network designer and is set at network deployment. Standby uses very little power. This period is determined by the necessary frequency of broadcasts. Following the idle stage, the Service Cycle will repeat.

\section{SimULATION OF Trim}

In order to test the TriM protocol, a variety of scenarios were simulated. The AweSim simulation software [13] using inserts coded in the $\mathrm{C}$ programming language to describe network behavior was used for this simulation study. AweSim is a general-purpose simulation tool that provides discrete event simulation of user defined networks [12]. The deployment of a MANET and the execution of the proposed MANET data communication protocol can be defined as a set of discrete events that occur during the operation of the protocol. Nine simulation scenarios were run using the benchmark described in [3].

The simulation runs vary data push parameters and data pull parameters. Three data broadcast sizes (50, 100 and 200 items) are simulated for each of the data pull settings. These broadcast sizes are referred to in the results as small, medium and large broadcasts, respectively. The data pull parameters are the frequency of data query and peer messaging. Data query and peer messaging are set to the same value. As both data query and peer message frequency are set to the same value, they are referred to collectively as pull frequency. The values used for pull frequency are 5, 20 and 40 items/sec. These are referred to as low, medium and high pull frequency, respectively. This variation simulates different loads on the network. This results in nine different workloads for each of the three scenarios. Each of the nine workloads was simulated 10 times. Table I shows basic parameters used in the simulation. The simulations assume that the servers and clients are initialized in a single staging area and then roam at random. However, the random speed must be within the mobility range and roaming region specified.

As with any simulation, some assumptions must be made. It is assumed that each broadcast transmission is equally split between static and dynamic data items. This means that a broadcast is always at least half full. It is further assumed that a client listens to the static portion of one broadcast transmission and to the entire dynamic portion of each broadcast transmission in its region. In data pull we assume a static number of data queries and peer messages per node. During simulation, the distance between nodes is calculated and compared to benchmark transmission ranges to determine if a client can hear a server and if a client transmission can reach other nodes. It is assumed that a client will send all data queries and routing requests to the closest server detected during synchronization if it is within client transmission range.

TABLE I

PARAmeters for Manet Data Communication Simulation

\begin{tabular}{|c|c|c|c|}
\hline Parameter & Value & Parameter & Value \\
\hline $\begin{array}{c}\text { Bandwidth } \\
\text { Server } \\
\text { Client } \\
\end{array}$ & $\begin{array}{l}2 \mathrm{Mbps} \\
100 \mathrm{Kbps}\end{array}$ & $\begin{array}{l}\text { Server Power Dissipation } \\
\text { Transmit Mode } \\
\text { Receive Mode } \\
\text { Standby Mode } \\
\end{array}$ & $\begin{array}{l}170 w \\
20 w \\
2 w\end{array}$ \\
\hline $\begin{array}{l}\text { Communication } \\
\text { Radius } \\
\text { Server } \\
\text { Client } \\
\end{array}$ & $\begin{array}{l}250 \text { meters } \\
100 \text { meters }\end{array}$ & $\begin{array}{l}\text { Client Power Dissipation } \\
\text { Transmit Mode } \\
\text { Receive Mode } \\
\text { Standby Mode } \\
\end{array}$ & $\begin{array}{l}7 \mathrm{w} \\
1 \mathrm{w} \\
0.1 \mathrm{w}\end{array}$ \\
\hline $\begin{array}{c}\text { CPU Power } \\
\text { Server } \\
\text { Client } \\
\end{array}$ & $\begin{array}{l}1700 \text { MIPS } \\
100 \text { MIPS }\end{array}$ & Mobility - all nodes & 0 to $20 \mathrm{~m} / \mathrm{sec}$ \\
\hline $\begin{array}{l}\text { Number of Nodes } \\
\text { Server } \\
\text { Client }\end{array}$ & $\begin{array}{l}20 \\
1000\end{array}$ & $\begin{array}{l}\text { Size of roaming region } \\
\text { Simulation Time }\end{array}$ & $\begin{array}{l}10 \mathrm{~km} \times 15 \mathrm{~km} \\
1 \text { hour }\end{array}$ \\
\hline
\end{tabular}

\section{A. Evaluation Criteria}

The evaluation criteria follow the benchmark described in [3]. They are:

Average Power Consumption The average power consumed by clients and the average power consumed by servers are calculated. For each client and server the power consumed per time unit is calculated by multiplying the percentage of time a node spends in each mode by the cost in power dissipation of each power mode.

Percentage of Coverage The effect of mobility that we measure is the percentage of clients out of range of all data broadcast transmissions. This demonstrates the effect of network mobility and implies the level of node disconnection.

Broadcast Effectiveness The broadcast portion of the MANET is important, as data push is energy efficient. The measure for this portion of data communication will be broadcast effectiveness, which is the ratio of items of interest in a broadcast to the total number of items transmitted.

Query Efficiency The data pull section will rely on the measurement of query efficiency. This is a measure of the percentage of data queries that get served during an entire simulation.

Peer Efficiency Peer-to-peer communication is a time when clients can communicate directly with clients. Peer efficiency is measured as a percentage of the messages sent to peers by the number of messages received by peers.

\section{B. Simulation Results}

The data presented is for the 9 workloads described above. It should be noted that when a server must choose 
between routing peer messages and serving data queries, routing takes precedence. The rationale is that data queries can be added to the next data broadcast while peer messages are dropped at the end of data pull. Figure 6 shows the average server power consumption simulation results for all 9 variations of broadcast size and pull frequency. Figures 7, 8, 9, 10, and 11 show the average client power consumption and the percentage of clients hearing a broadcast, Broadcast Effectiveness, Query Effectiveness and Peer Effectiveness, respectively.

To understand the results, it is important to know that the length of each stage of the service cycle changes from one workload to the next. For instance, as the maximum size of the data broadcast increases, so does the length of the data push stage. When the pull frequency increases, the data push stage also increases in length. As the length of the service cycle increases because of a larger data broadcast, the average power consumption for server decreases as less time is spent transmitting. While each broadcast transmission is longer, the amount of time waiting for the other servers to transmit also increases. As the length of a service cycle increases due to a larger pull frequency, the average power consumption increases due to the increase of transmission by each server. A larger pull frequency requires a greater number of data queries to be processed per second.

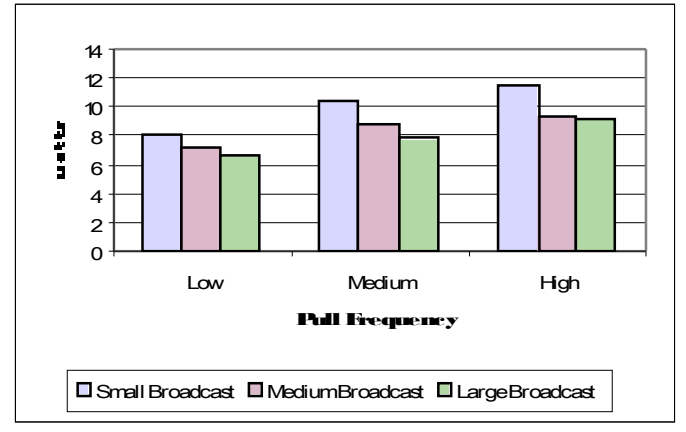

Fig. 6. Avg. Server Power Consumption

The average power consumption for clients is universally low, between receive and standby. This is the result of the high level of disconnection that occurs as the nodes move randomly in a very large area. As a large percentage of clients do not hear a server during synchronization, many clients spend a large percentage of their time in standby.

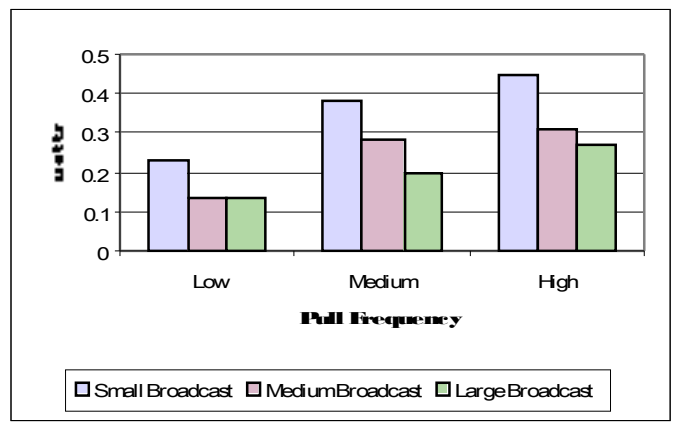

Fig. 7. Avg. Client Power Consumption
As the length of the service cycle increases because of a longer data pull corresponding to the larger data broadcast, the average power consumption for server decreases as less time is spent transmitting. While each node transmits longer, the amount of time waiting for all of the other nodes to transmit also increases. As the length of a service cycle increases due to larger data push caused by a larger pull frequency, the average power consumption increases due to the increase of transmission by each server. A larger pull frequency requires a greater number of data queries to be processed per unit time. Overall, the average power consumption for servers is near the level for receive mode. By infrequently transmitting, power consumption is kept low. Servers spend most of their life in receive mode, waiting to serve data queries and routing requests. As the number of clients served by a server increases, the amount of time spent transmitting will also increase.

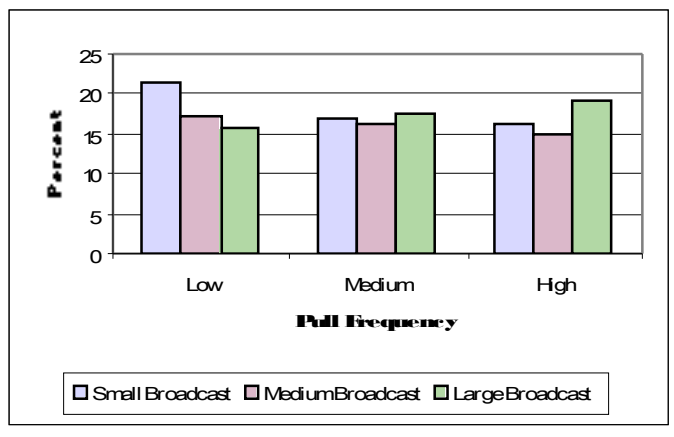

Fig. 8. \% of Clients hearing Data Broadcast

The number of clients hearing a data broadcast is low. The number changes a small percent from one workload to another. The large size of the region, the random movement of servers and clients and the small number of servers makes the possibility of hearing a broadcast rather small.

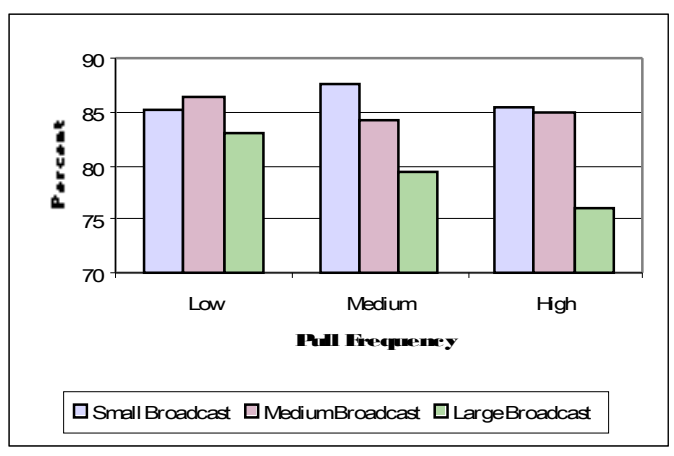

Fig. 9. Broadcast Effectiveness

In Figure 8 we see the simulated broadcast effectiveness. As expected, this number is very high. As the likelihood of a client hearing more than a single data broadcast is very small, the clients will not hear multiple static portions of broadcasts. The broadcast effectiveness shown does decrease a small amount. As the pull frequency increases, the ability of a server to handle all data queries and peer message routing requests decreases.

The query efficiency and peer efficiency are both very low. The query efficiency is the lower of the two. This can be explained due to the large percentage of clients that are 
not near to a server. While 15 to $20 \%$ can hear a server data broadcast, the transmission radius of the client would mean that even fewer clients can be heard from a server for data query and message routing service. It should be noted that peer efficiency is much better. With the large number of clients in this scenario (1000) compared to servers (20), a client is far more likely to be near a client with which to message. In addition, the simulation has a preference for peer message routing over query service when both are needed. Query efficiency is shown in Figure 10 and peer efficiency in Figure 11.

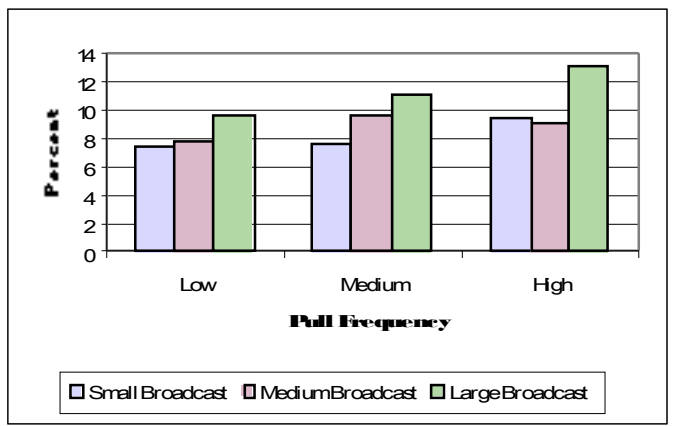

Fig. 10. Query Efficiency

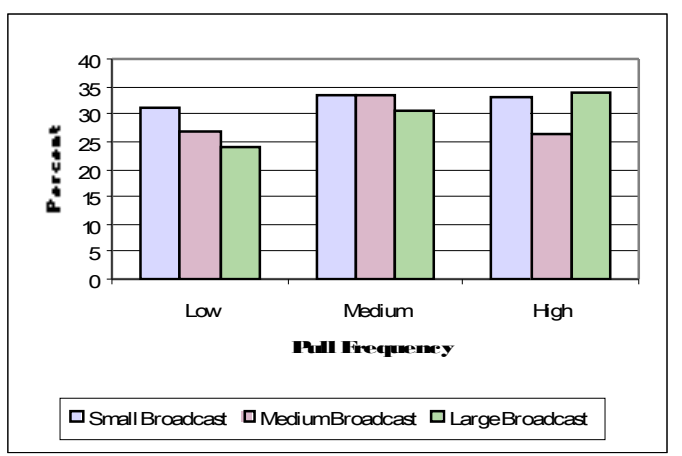

Fig. 11. Peer Efficiency

\section{CONCLUSIONS AND FUTURE WORK}

This paper presented a protocol that allows all three forms of MANET data communication, preserving power and accommodating limited bandwidth and mobility. The proposed protocol, TriM, has been simulated previously in a small $1 \mathrm{~km} \times 1 \mathrm{~km}$ region [5]. This paper extends that work by evaluating TriM in the larger $10 \mathrm{~km} \mathrm{x} 15 \mathrm{~km}$ region of the military scenario. The TriM protocol appears capable of proving all three forms of data communication available in a MANET within the restrictions of a wireless mobile network. This capability extends to regions of different sizes.

Future work will consider additional MANET scenarios. The effect of increased transmission ranges should also be investigated. The ability to increase coverage through data relay and greater node cooperation should be studied. A New benchmark, proposed in [3] is used to set up the architecture and workload of the MANET simulated. The benchmark criteria for evaluation are also used. Further work on the protocol itself is in order. Adding real-time capabilities, directional antennas, variable power transmissions, etc. provides a list of items that can be added to a new or modified protocol. Finally, consideration of additional mobility models is recommended.

\section{REFERENCES}

[1] Das, S., Castañeda, R., and Yan, J., "Simulation-Based Performance Evaluation of Routing Protocols for Mobile Ad Hoc Networks," Mobile Networks and Applications, 5(3): pp. 179-189, 2000.

[2] Datta, A., VanderMeer, D., Kim, J., Celik, A., and Kumar, V., "Adaptive Broadcast Protocols to Support Efficient and Energy Conserving Retrieval from Databases in Mobile Computing Environments." A TimeCenter Technical Report (University of Arizona), 1997.

[3] Fife, L. and Gruenwald, L. "A Mobile Ad-Hoc Network Data Communication Benchmark," In Proc. $18^{\text {th }}$ International Conference on Parallel and Distributed Computing Systems, Las Vegas, Nevada, USA, September 2005.

[4] Fife, L. and Gruenwald, L., "Research Issues for Data Communication in Mobile Ad-Hoc Network Database Systems," ACM SIGMOD Record, 32(2): June 2003.

[5] Fife, L. and Gruenwald, L., TriM: Tri-Modal Data Communication in Mobile Ad-Hoc Networks. In Proc. 15 $5^{\text {th }}$ International Conference on Database and Expert System Applications (DEXA 2004), Zaragoza. Spain, August/September 2004 (LNCS 3180).

[6] Fife, L., "TriM: Tri-Modal Data Communication in Mobile Ad-Hoc Network Database Systems," Dissertation, University of Oklahoma, 2003.

[7] Gruenwald, L., Javed, M., and Gu, M., "Energy-Efficient Data Broadcasting in Mobile Ad-Hoc Networks," In Proc. International Database Engineering and Applications Symposium (IDEAS '02), Edmonton, Canada, July 2002.

[8] Guo, Y., Pinotti, M., and Das, S., "A New Hybrid Broadcast Scheduling Algorithm for Asymmetric Communication Systems," ACM Mobile Computing and Communications Review, 5(4): pp. 3954, 2001.

[9] Johansson, P., Larsson, L., Hedman, N., Mielczarek, B., and Degermark, M., "Scenario-based Performance Analysis of Routing Protocols for Mobile Ad-hoc Networks," In Proc. $5^{\text {th }}$ International Conference on Mobile Computing and Networking (MOBICOM'99), Seattle, Washington, USA, August 1999.

[10] Jones, C., Sivalingam, K., Agrawal, P., and Chen, J., "A Survey of Energy Efficient Protocols for Wireless Networks," Wireless Networks, 7 pp. 343-358, 2001

[11] Jung, I., You, Y., Lee, J., and Kim, K. Broadcasting and Caching Policies for Location-Dependent Queries in Urban Environments. In Proc. Second International Symposium on Mobile Commerce (WMC '02), pp. 54-60, Atlanta, Georgia, USA, September, 2002.

[12] O'Reilly, J. and Lilegdon, W. Introduction to AweSim. In Proc. 1999 Winter Simulation Conference, pp. 196-200, 1999

[13] Pritsker, A. and O'Reilly, J. Simulation with Visual SLAM and AweSim, $2^{\text {nd }}$ ed. New York: John Wiley \& Sons, 1999.

[14] Tang, C., Raghavendra, A., and Prasanna, V. Energy Efficient Adaptation of Multicast Protocols in Power Controlled Wireless Ad Hoc Networks. In Proc. International Symposium on Parallel Architectures, Algorithms and Networks (ISPAN02), Manila, Phillipines, May, 2002.

[15] Tseng, Y., Ni, S., Chen, Y., and Shue, J., "The Broadcast Storm Problem in a Mobile Ad Hoc Network," Wireless Networks, 8 pp. 153-167, 2002

[16] Wieselthier, J., Nguyen, G., and Ephremides, A., "Algorithms for Energy-Efficient Multicasting in Static Ad Hoc Wireless Networks," Mobile Networks and Applications, 6(4): pp. 251-263, 2001.

[17] Wieselthier, J., Nguyen, G., and Ephremides, A. Resource-Limited Energy-Efficient Wireless Multicast of Session Traffic. In Proc. 34th Hawaii International Conference on System Sciences (HICSS34), Wailea, Maui, Hawaii, USA, January 2001.

[18] Williams, B. and Camp, T., "Comparison of Broadcasting Techniques for Mobile Ad Hoc Networks," In Proc. International Symposium on Mobile Ad Hoc Networking and Computing (MOBIHOC'02), Lausanne, Switzerland, June 2002. 